\title{
Influence of photon energy on conductivity of photoconductive semiconductor switches fabricated from semi-insulating GaP
}

K. Piwowarski, M. Suproniuk, P. Kamiński, B. Perka, R. Kozłowski, et al.

K. Piwowarski, M. Suproniuk, P. Kamiński, B. Perka, R. Kozłowski, M. Teodorczyk, "Influence of photon energy on conductivity of photoconductive semiconductor switches fabricated from semi-insulating GaP," Proc. SPIE 11442, Radioelectronic Systems Conference 2019, 114420M (11 February 2020); doi: 10.1117/12.2565269

SPE. Event: Radioelectronic Systems Conference 2019, 2019, Jachranka, Poland 


\title{
Influence of photon energy on conductivity of photoconductive semiconductor switches fabricated from semi-insulating GaP
}

\author{
K. Piwowarski*a , M. Suproniuk ${ }^{\mathrm{a}}$, P. Kamiński ${ }^{\mathrm{b}}$, B. Perka ${ }^{\mathrm{a}}$, R. Kozłowski ${ }^{\mathrm{b}}$, M. Teodorczyk $^{\mathrm{b}}$ \\ ${ }^{a}$ Military University of Technology, gen. Sylwestra Kaliskiego 2, Warsaw, 00-908, Poland; \\ binstitute of Electronic Materials Technology, Wolczynska 133, Warsaw, 01-919, Poland
}

\begin{abstract}
The article presents the experimental results of research works aimed at manufacturing photoconductive semiconductor switches (PCSSs) on semi-insulating (SI) gallium phosphide (GaP) wafers. The PCSS is an electrical switch, triggered into the conduction state by means of an optical pulse with specific photon energy. The switches presented in the paper are developed for the applications in power systems and high-pulse voltage generators. The paper presents time courses of the photocurrent being a response to the excitation of semiconductor material with an optical pulse that generates excess charge carriers. The effects of laser beam photon energy on the height and time constant of photocurrent pulses were determined. The results of measurements of photocurrent in the conduction state for various values of electric field strength are presented. The research methodology was discussed and the measuring system used was described. Possible directions of further research were also presented. The research was carried out thanks to the cooperation of scientific teams from the Military University of Technology (WAT) and the Institute of Electronic Materials Technology (ITME).
\end{abstract}

Keywords: semi-insulating GaP, photoconductive semiconductor switches, impulse systems, switch characteristics.

\section{INTRODUCTION}

Photoconductive semiconductor switch (PCSS) is an electric switch characterized by high voltage durability. Application of PCSS switches allows theoretically to deliver tens of megawatts of power in the order of nanoseconds. Literature studies have shown that these devices can work in the blocking state with a voltages up to $100 \mathrm{kV}$ [1]. In addition, the conducted experiments showed the possibility of conducting currents of approximately $1 \mathrm{kA}$ [2]. The transition from blocking to conduction state, occurs through the use of an optical pulse with specific photon energy, which results in galvanic separation of the switch operation and control systems. The experiments carried out so far show that this switches can operate with repetition rate of $1 \mathrm{kHz}[1-3]$.

Currently, the possibilities of using PCSS switches in impulse systems and in the power industry to control the transmission of electricity are being investigated. Pulse systems are commonly used in the automation of electronic systems control and in military systems, such as pulsed lasers and microwave sources. Their task is to transfer high peak power during a single pulse. Traditional pulse systems mainly use spark gap switches. In power industry, mechanical switches are used for energy transmission. Both spark gap and mechanical switches allow the system to operate at voltages greater than $100 \mathrm{kV}$ and currents above $100 \mathrm{~A}$. The significant disadvantages of spark gab switches are the low pulse repetition rate, the inability to precisely control the moment of switching, as well as the short time of possible operation of the device. The main problem with mechanical switches are the large spread of the switching-on time value and the possibility of an electric arc during switching $[4,5]$. Photoconductive semiconductor switches do not have these disadvantages.

A typical PCSS is made of semiconductor material with very high resistivity and a thickness of 0.5 to $1 \mathrm{~mm}$, on the surface of which electrodes are placed for connection to the electrical system. An overview of the basic PCSS constructions was previously described in [6]. On its basis, the solution used in the research was selected, shown in figure 1.

*karol.piwowarski@wat.edu.pl

Radioelectronic Systems Conference 2019, edited by Piotr Kaniewski, Jan Matuszewski, Proc. of SPIE Vol. 11442, 114420M · @ 2020 SPIE · CCC code: 0277-786X/20/\$21 · doi: 10.1117/12.2565269 


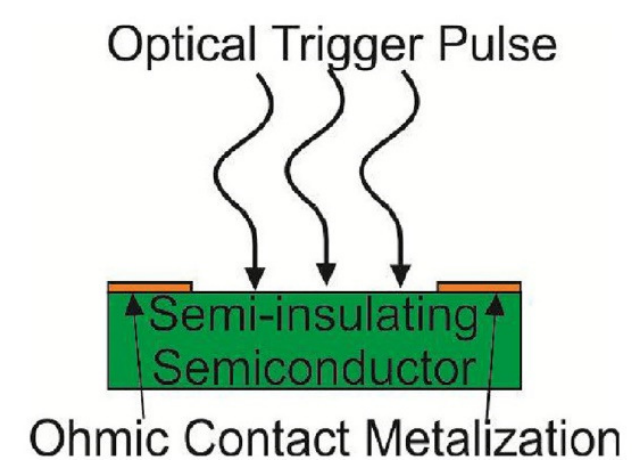

Figure 1. Scheme of the photoconductive semiconductor switch used for the tests carried out in this work.

One of the semiconductor materials used to build photoconductive semiconductor switches is semi-insulating (SI) GaAs. The switches made of it, operating in a non-linear mode, are able to block voltages of $40 \mathrm{kV}$ and conduct current up to 400 A $[1,2,7]$. Semiconductor materials with a wider energy gap than GaAs of $1.424 \mathrm{eV}$ should theoretically achieve better performance. They are, among others, SI GaP, SI 6H-SiC, SI 4H-SiC and SI GaN with a band gap width of 2.26, $3.0,3.23$ and $3.39 \mathrm{eV}$ respectively at $300 \mathrm{~K}[6,8]$. Research confirms better properties of PCSS made of SI 6H-SiC, SI $4 \mathrm{H}-\mathrm{SiC}$ and SI GaN compared to switches made of SI GaAs [8, 9].

PCSS switches can work in linear (conventional) and non-linear (avalanche) modes. In the first of them, the absorption of one photon generates the formation of one electron-hole pair. When the light source is switched off, the excess charge carriers recombine, which causes the semiconductor material properties to return to the state before lighting. In the case of photon energy greater than the width of the forbidden gap $\left(h v>E_{g}\right)$, excess charge carriers are generated mainly in the surface area and in order to obtain a sufficiently low switch resistance, the optical energy density should be in the order of several $\mathrm{kJ} / \mathrm{cm}^{2}$. As a result, this type of switch is most often constructed with a lateral electrode system. In the case of absorption of photons with energy smaller than the width of the energy gap $\left(h v<E_{g}\right)$, the generation of excess charge carriers occurs from defective levels and the speed of their generation depends on the location of these levels in the energy gap. Depending on the properties of the donor or acceptor defect centers, photons can penetrate the semiconductor to a depth of several millimeters. Because the activation in the linear mode is independent of the electric field value in the switch, operation in this mode is only possible in low voltage systems with an electric field strength not exceeding $4 \mathrm{kV} / \mathrm{cm}$. As a result of reduced current density, switches operating in linear mode are characterized by longer life time [2].

Work in non-linear mode, also called avalanche mode, is carried out at much higher electric field intensities than in linear mode. Excited as a result of lighting, excess charge carriers receive additional energy as a result of a strong electric field and impact ionization, which occurs by knocking out valence electrons and electrons from defect centers to the conduction band. Thus, in this mode a single photon generates many charge carriers. After initiation, the avalanche process of charge carrier multiplication is maintained until the electric field strength in the switch drops below the appropriate value, depending on the resistance of the external system. In non-linear mode, lower optical energy density can be used because the laser beam is only used to trigger the process of impact ionization [10].

\section{EXPERIMENTAL RESULTS}

\subsection{Description of samples}

Test switches made of semi-isolating GaP single crystals (SI GaP) marked with labels GaP-1 and GaP-2 were used in the studies. On the polished surface of the SI GaP plates, two ohmic contacts were made by applying gold at a distance of $2 \mathrm{~mm}$ from each other, to which two electrodes from electrolytic copper were attached. SI GaP plates were in the form of chips with an area of $1 \times 1 \mathrm{~cm}^{2}$ and a thickness of $0.5 \mathrm{~mm}$. Before attaching the electrodes, the surface of the material was mechanically and chemically cleaned. The electrodes were made in the form of strips $0.3 \mathrm{~mm}$ thick, $10 \mathrm{~mm}$ wide and $15 \mathrm{~mm}$ long, with $0.2 \mathrm{~mm}$ thick, $2 \mathrm{~mm}$ wide and $100 \mathrm{~mm}$ long leads. Both electrodes and leads were covered with a layer of silver. To ensure protection against external factors and reduce the speed of surface recombination of excess charge carriers, after connecting the electrodes, the switches were coated with a $200 \mathrm{~nm} \mathrm{SiO}$ layer and then embedded in a transparent resin. Figure 2 illustrates the appearance of the test switches, ready for measurement. 


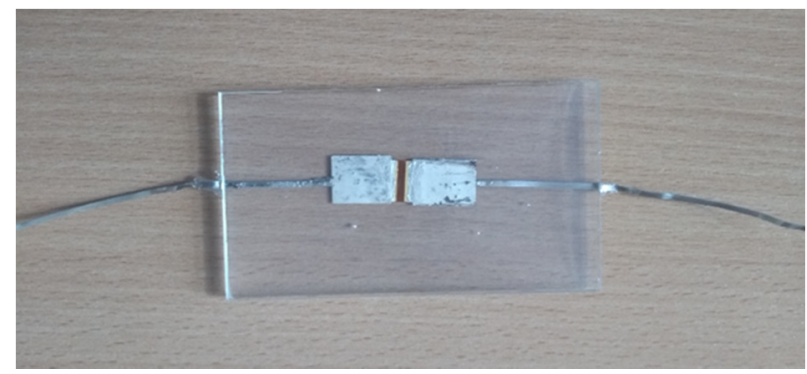

Figure 2. Photo of investigated PCSS switches made of semi-insulating gallium phosphide.

\subsection{Dark current measurements}

The measurements carried out are a continuation of the research described in [11]. In the blocking state, the METREL MI $3210 \mathrm{TeraOhm}$ XA meter was used to measure switch parameters. It served as a source of DC voltage and a meter of resistance and current. It allows current measurements in the range from $0.1 \mathrm{nA}$ to $5 \mathrm{~mA}$ at voltages from $50 \mathrm{~V}$ to $10 \mathrm{kV}$. For each sample, for every measured value starting from a voltage of $500 \mathrm{~V}$ with a step of $500 \mathrm{~V}$, a series of three measurements were made. The obtained results of current measurements depending on the voltage and resistance as a function of electric field strength are presented in figures 3 and 4 respectively.

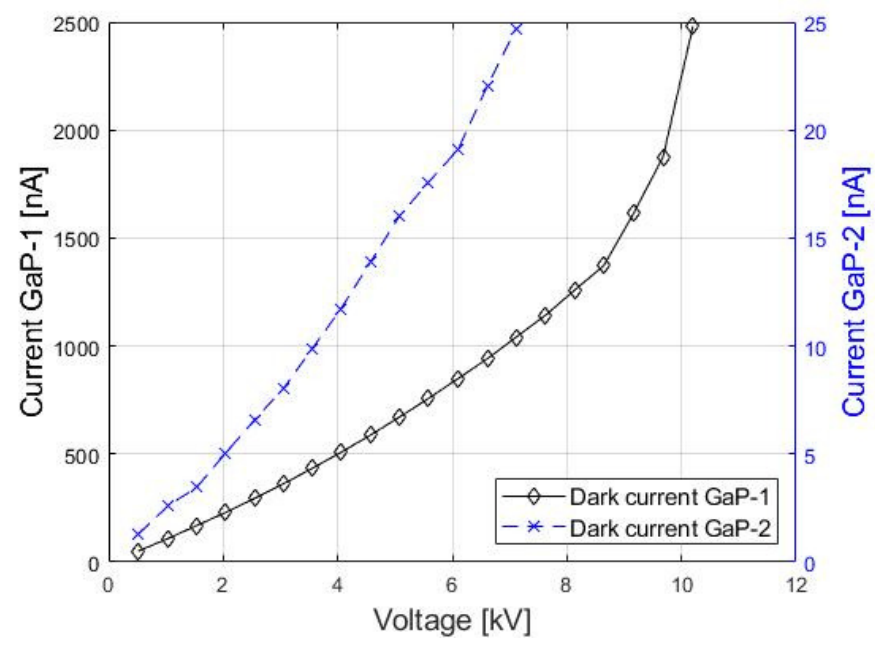

Figure 3. Current-voltage characteristics of test switches made of semi-insulating gallium phosphide measured at $300 \mathrm{~K}$. 


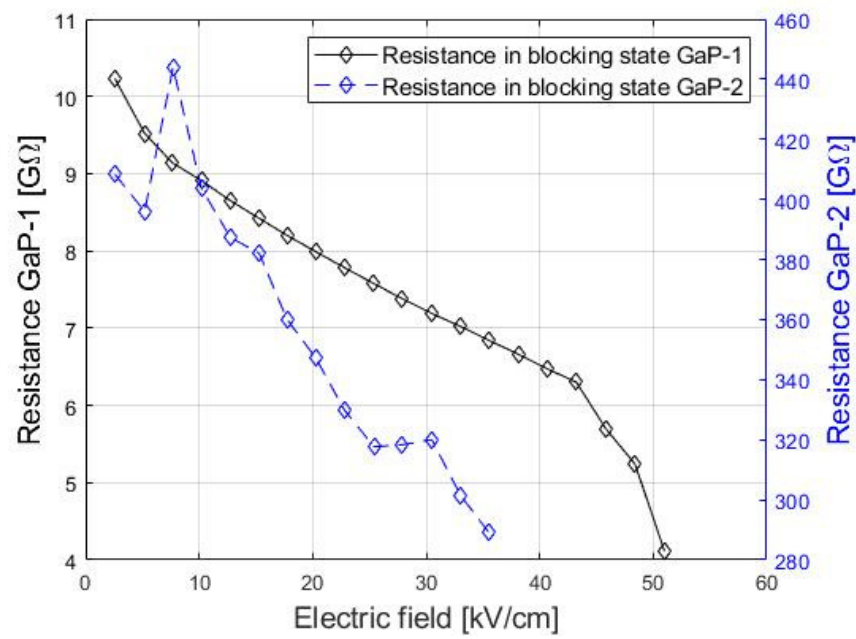

Figure 4. Dependence of switch resistance on the electric field strength in the active area, determined on the basis of dark current intensity measurements at a temperature of $300 \mathrm{~K}$.

Analyzing the characteristics shown in figures 3 and 4, it can be seen that the current conducted through the GaP-1 sample is 2 orders of magnitude larger than in the case of $\mathrm{GaP}-2$, and the difference of samples resistance values is also in range of 2 orders of magnitude. The dark current of the GaP-2 sample in the whole tested range has a linear course, while the sample resistance decreases linearly in the range from 7.7 to $35.55 \mathrm{kV} / \mathrm{cm}$ (corresponding to voltages from 3.46 to 7.11 $\mathrm{kV})$. GaP-2 measurement results in the range from 2.58 to $7.7 \mathrm{kV} / \mathrm{cm}$ can be explained by the inaccuracy of the measuring device occurring at low system voltages. In the voltage range from $516 \mathrm{~V}$ to $8.65 \mathrm{kV}$, corresponding to the electric field strength from 2.58 to $43.23 \mathrm{kV} / \mathrm{cm}$, linear characteristics of dark current waveforms and blocking resistance for the GaP1 sample were obtained. In the voltage range from 8.65 to $10.2 \mathrm{kV}(43.23$ to $51 \mathrm{kV} / \mathrm{cm})$ there is a significant increase in the value of dark current from 1373 to $2480 \mathrm{nA}$ and a decrease in resistance from 6.3 to $4.11 \mathrm{G} \Omega$. Despite the non-linearity of the characteristic curves in the voltage range shown and the corresponding electric field strengths, the GaP-1 sample shows a blocking resistance of more than $1 \mathrm{G} \Omega$, which is a satisfactory result.

The conductivity in the blocking state $\sigma_{\mathrm{o}}$ is expressed by the formula:

$$
\sigma_{0}=\frac{d}{R s}
$$

In which $d$ is the width of the semiconductor area between the electrodes, $R$ - the resistance determined in the measurements and $s$ is the cross-sectional area of the active region of the sample. The conductivity of the samples varied from $1.96 \cdot 10^{-7}$ to $4.87 \cdot 10^{-7} \Omega^{-1} \mathrm{~cm}^{-1}$ for GaP-1 and from $4.51 \cdot 10^{-9}$ to $6.92 \cdot 10^{-9} \Omega^{-1} \mathrm{~cm}^{-1}$ for GaP-2. Figure 5 shows the calculated conductivity values of the tested samples in the blocking state. 


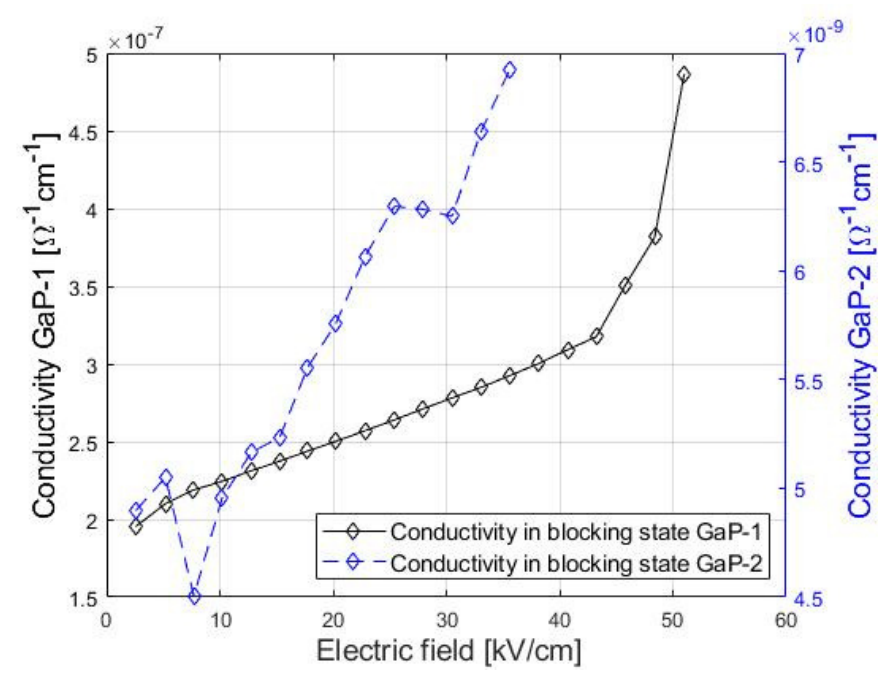

Figure 5. Conductivity of test switches with SI GaP in a blocking state depending on the electric field strength at $300 \mathrm{~K}$

\subsection{Photocurrent measurements}

Measurements in the conduction state were made in a system consisting of the METREL MI 3210 TeraOhm XA meter, which was a voltage source, and connected in series studied PCSS switch and resistor with known resistance. Voltage drops on the switch and resistor, as well as the response of the system to the impulse switching the sample into the conduction state were recorded using an oscilloscope. The optical switching pulse, with a width of $\sim 5 \mathrm{~ns}$, was generated by a tunable laser, and the laser beam was emitted with a frequency of $10 \mathrm{~Hz}$. Time courses of voltage drop across the resistor were recorded after averaging 128 waveforms caused by the optical pulse. The schematic diagram of the constructed measurement system is shown in figure 6.

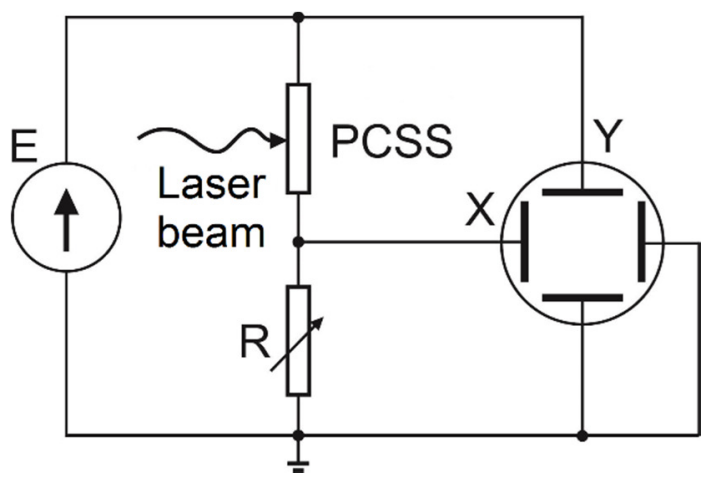

Figure 6. Schematic diagram of the measurement system used to measure the test characteristics of SI GaP switch in the conduction state.

The GaP-1 sample was tested in the wavelength range from 410 to $710 \mathrm{~nm}$, corresponding to photon energy in the range from 1.75 to $3.0 \mathrm{eV}$, with a laser beam energy of $190 \mu \mathrm{J}$ and an applied voltage of $10.2 \mathrm{kV}$. The maximum achieved values of the conducted current depending on the photon energy for the GaP-1 switch are shown in figure 7. 


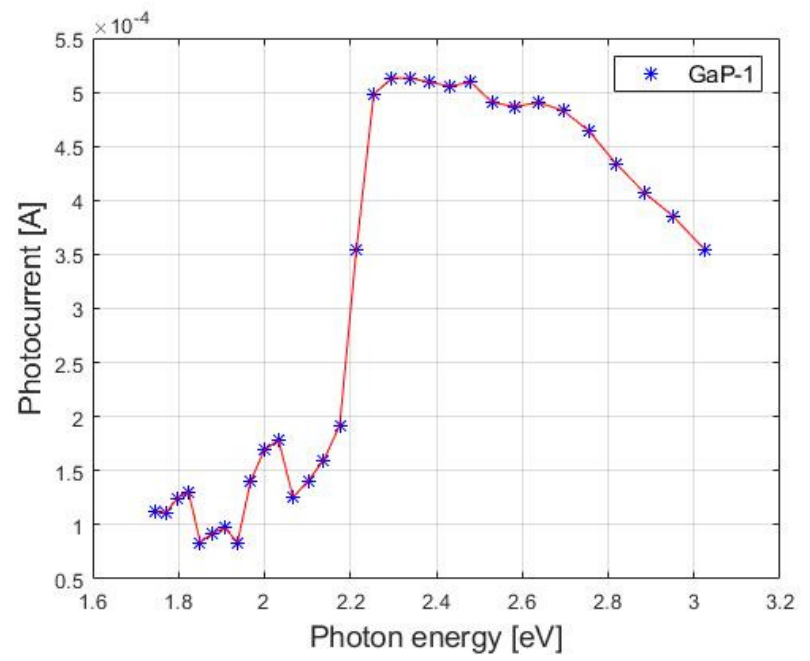

Figure 7. Maximum photocurrent values achieved for the GaP-1 sample in the conduction state for a $10.2 \mathrm{kV}$ system voltage at $300 \mathrm{~K}$. Laser beam energy $60 \mu \mathrm{J}$.

In figure 7, a 2.5 times increase in conducted photocurrent can be observed, from 0.2 to $0.5 \mathrm{~mA}$, for photon energy in the range 2.18 to $2.25 \mathrm{eV}$. This increase is associated with approaching the absorption edge, occurring at energy close to $2.26 \mathrm{eV}$, the width of band gap of gallium phosphide. In conduction of current generated by photons with lower energy than that, participate only charge carriers generated using energy levels of deep defect centers in material. Only the charge carriers, generated through the energy levels of deep defect centers in the material, participate in the conductivity of the current generated with lower photon energy.

For the GaP-2 sample, a series of tests were carried out for two selected wavelengths of the laser beam in the range of applied voltages from $1 \mathrm{kV}$ to $7 \mathrm{kV}$. The energy of the laser beam in the measurement series was $60 \mu \mathrm{J}$. The wavelengths were $450 \mathrm{~nm}$ and $700 \mathrm{~nm}$, which corresponds to photon energy of 2.76 and $1.77 \mathrm{eV}$, respectively. The maximum achieved values of the conducted current through the GaP-2 switch as a function of the applied voltage are shown in figure 8 .

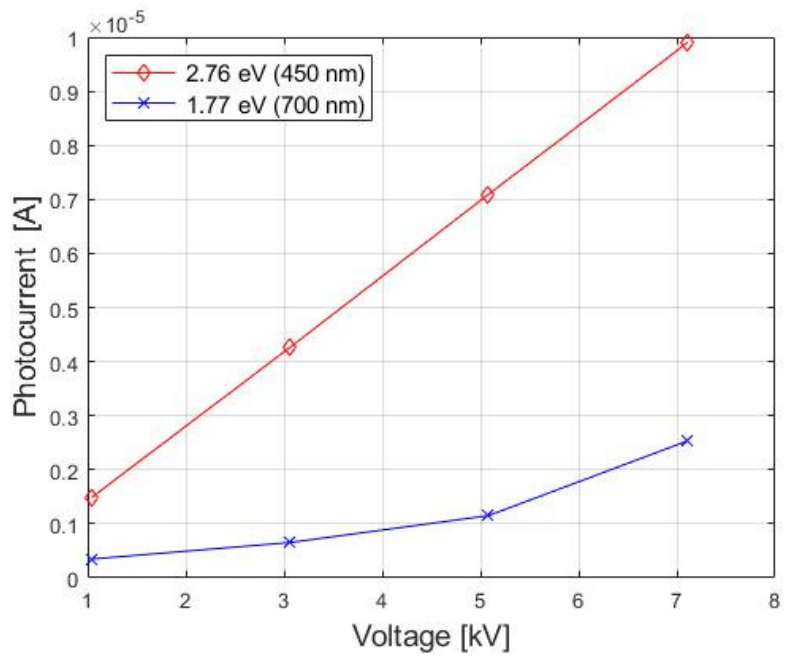

Figure 8. Maximum photocurrent values for a GaP-2 sample at a given photon energy depending on the applied voltage measured in a conduction state at $300 \mathrm{~K}$. Laser beam energy $60 \mu \mathrm{J}$. 
Following the obtained characteristics waveforms in figure 8, it can be seen that the photocurrent intensity increases linearly with the increase of the applied voltage for photon energy greater than the energy corresponding to the absorption edge $\left(h v>E_{g}\right)$. For photons with energy of $2.76 \mathrm{eV}$, there is a linear increase in conducted current with increasing voltage. Photocurrent intensity at $1.04 \mathrm{kV}$ and $7.11 \mathrm{kV}$ voltages are $1.49 \mu \mathrm{A}$ and $9.9 \mu \mathrm{A}$, respectively. Photocurrent intensity for $2.76 \mathrm{eV}$ photon energy is about 4 times higher than at the same voltage values for $1.77 \mathrm{eV}$ photon energy, $0.35 \mu \mathrm{A}$ and $2.53 \mu \mathrm{A}$, respectively.

Conductivity in the conduction state was determined based on Ohm's law and equation 1. Figures 9 and 10 show the results of conductivity calculations, respectively for the GaP-1 sample in the entire photon energy range tested, and for the GaP2 sample, for the photon energy value $1.77 \mathrm{eV}$ and $2.76 \mathrm{eV}$.

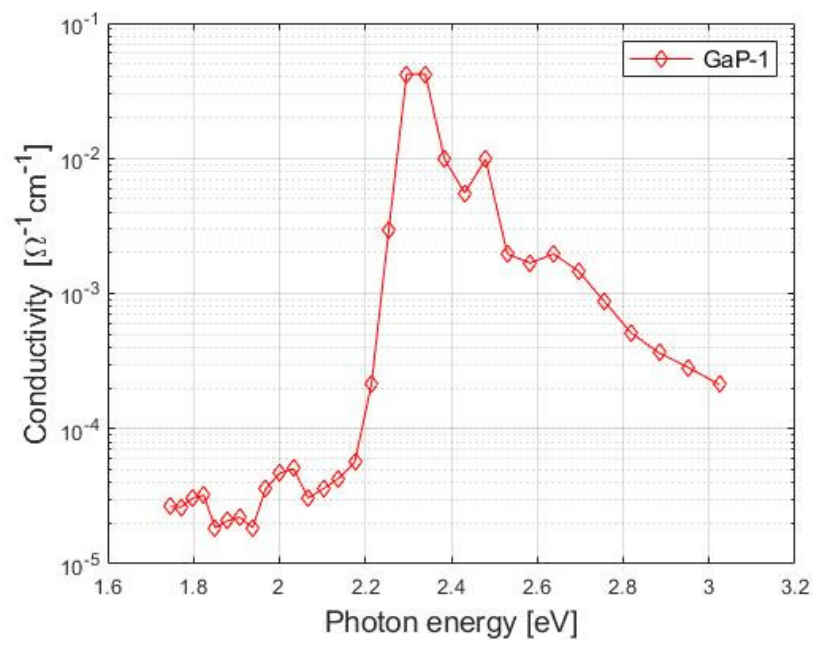

Figure 9. Dependence of conductivity on photon energy for the GaP-1 sample in temperature 300K. OY axis presented on a logarithmic scale.

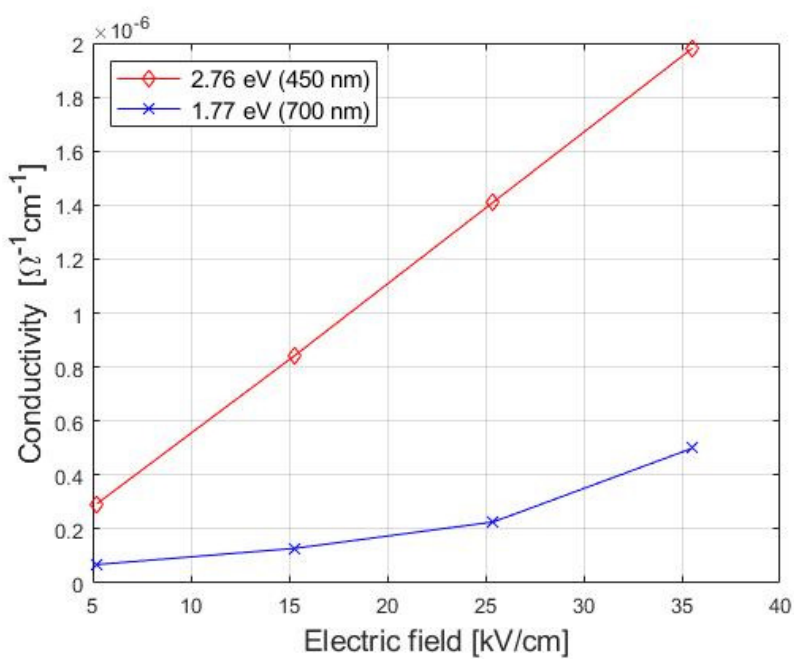

Figure 10. Dependence of conductivity on the electric field strength for the GaP-2 sample in temperature $300 \mathrm{~K}$. The red results indicate photon energy equal to $2.76 \mathrm{eV}$, the blue results indicate photon energy $1.77 \mathrm{eV}$.

Figure 9 shows an increase in the conductivity of the GaP-1 sample by 3 orders of magnitude for photon energy in the range of 2.18 to $2.3 \mathrm{eV}$. The maximum value of the conductivity achieved was $0.042 \Omega^{-1} \mathrm{~cm}^{-1}$ for a wavelength of $540 \mathrm{~nm}$ and $530 \mathrm{~nm}$, corresponding to the photon energy of $2.3 \mathrm{eV}$ and $2.34 \mathrm{eV}$. Figure 10 shows that the difference in conductivity 
for $1.77 \mathrm{eV}$ and $2.76 \mathrm{eV}$ photons was within 1 order of magnitude. At an electric field strength of $35.55 \mathrm{kV} / \mathrm{cm}$, values of $5.0 \cdot 10^{-7}$ and $1.98 \cdot 10^{-6} \Omega^{-1} \mathrm{~cm}^{-1}$ were achieved.

\subsection{Determination of time constants}

Time constants were determined for the recorded waveforms of voltage drop. They affect the switch off time and are related to the parameters of defect centers. The inverse of the time constant of these $e_{T}$ waveforms, determining the rate of thermal emission of charge carriers from defect centers, is described by the Arrhenius equation.

$$
e_{T}=A T^{2} \exp \left(-\frac{E_{a}}{k_{B} T}\right)
$$

In equation (2) $E_{a}$ means the activation energy of the centers, $A$ is the product of material constant $\gamma$ and capture cross section for electrons $\sigma, T$ is the temperature on an absolute scale, while $k_{B}$ is the Boltzmann constant.

These waveforms can be described by the sum of exponential functions and expressed by the equation in the form:

$$
i(t)=a_{1} \cdot e^{b_{1} \cdot x}+\ldots+a_{n} \cdot e^{b_{n} \cdot x}
$$

A single term of the sum represents course associated with one type of defect centers in the switch material. The coefficient $a$ is related to the concentration of charge carriers emitted from a given defect center. The coefficient $b$ is related to the parameters of the defect center. The methods of obtaining and analyzing defect centers parameters based on Arrhenius plot, were the subject of earlier studies presented in detail in $[12,13]$.

The recorded course of non-stationary photoconductivity illustrating the response of the system to the impulse switching the GaP-1 sample into the conduction state for $2.25 \mathrm{eV}$ photon energy at $10.2 \mathrm{kV}$ is shown in figure 11 . Figure 12 shows the response of a GaP-2 sample to an optical pulse of $2.76 \mathrm{eV}$ photon energy $7.11 \mathrm{kV}$.

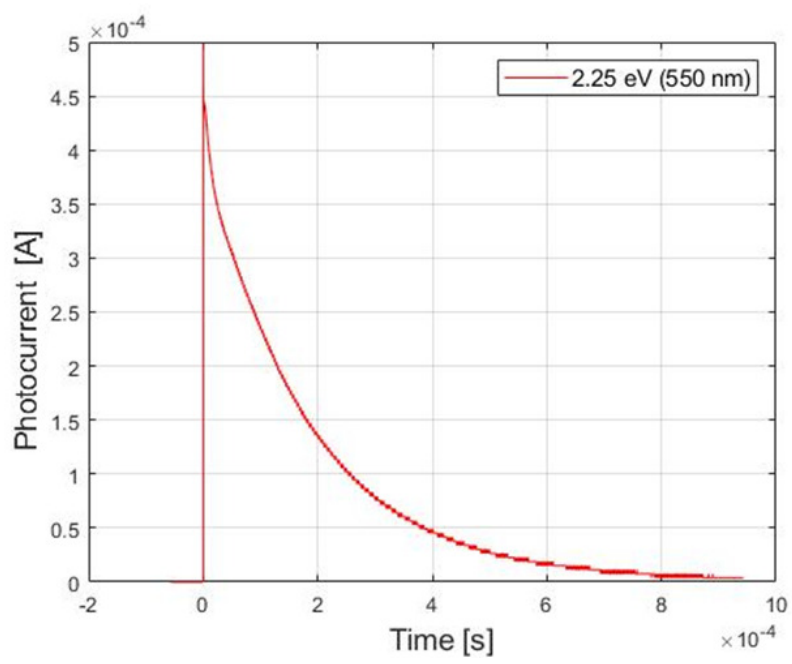

Figure 11. The obtained photocurrent waveform in the switch as a function of time at a temperature of $300 \mathrm{~K}$, at a beam energy of $190 \mu \mathrm{J}$. Supply voltage $10.2 \mathrm{kV}$. The moment of switching pulse is at the point $\mathrm{t}=0$. 


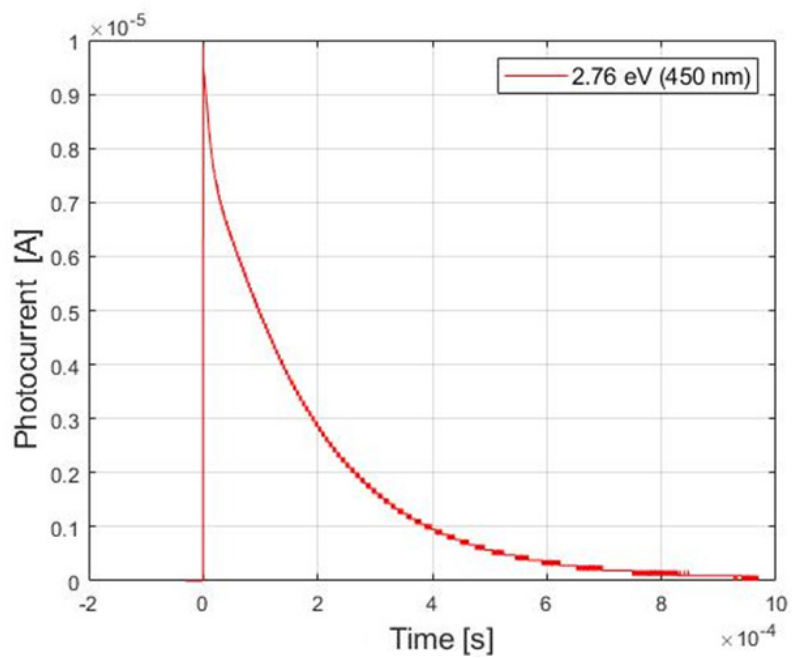

Figure 12. The obtained photocurrent waveform in the switch as a function of time at a temperature of $300 \mathrm{~K}$, at a beam energy of $60 \mu \mathrm{J}$. Supply voltage $7.11 \mathrm{kV}$. The moment of switching pulse is at the point $\mathrm{t}=0$.

Figures 13 and 14 show fragments of recorded waveforms of photocurrent flowing through the sample from the time the maximum was reached until the switch returned to the blocking state. For the GaP-1 sample, the results of measurements for $10.2 \mathrm{kV}$ voltage and $2.25 \mathrm{eV}$ photon energy $(550 \mathrm{~nm})$ are presented, while for the GaP-2 sample the results for $7.11 \mathrm{kV}$ voltage and $2.76 \mathrm{eV}(450 \mathrm{~nm})$ photon energy are illustrated.

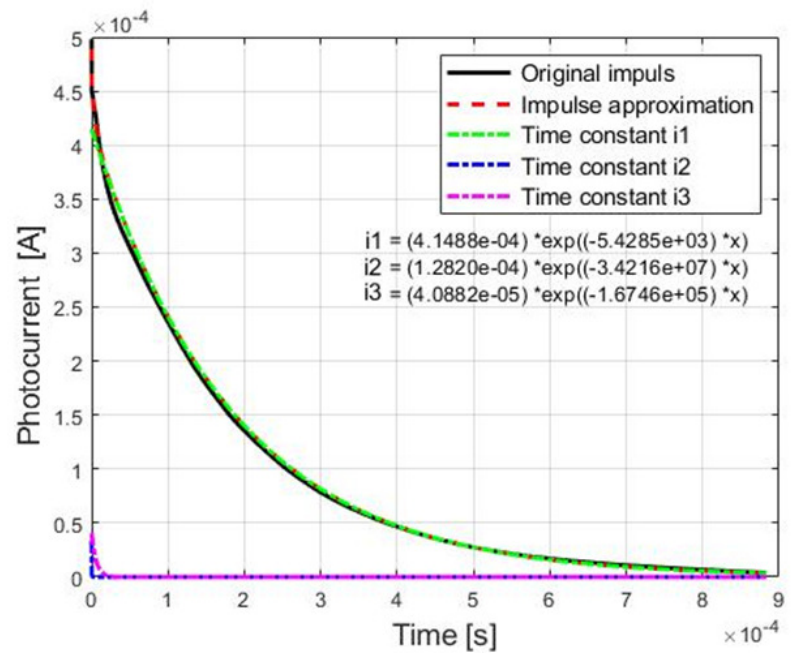

Figure 13. Determined time constants of the photocurrent pulse for the GaP-1 switch in the conduction state when excited with $2.25 \mathrm{eV}(550 \mathrm{~nm})$ photons at $10.2 \mathrm{kV}$. The original pulse waveform is marked in black, the numerically determined sum of time constants in red, as green, blue and purple are marked in order time constants i1, i2 and i3 


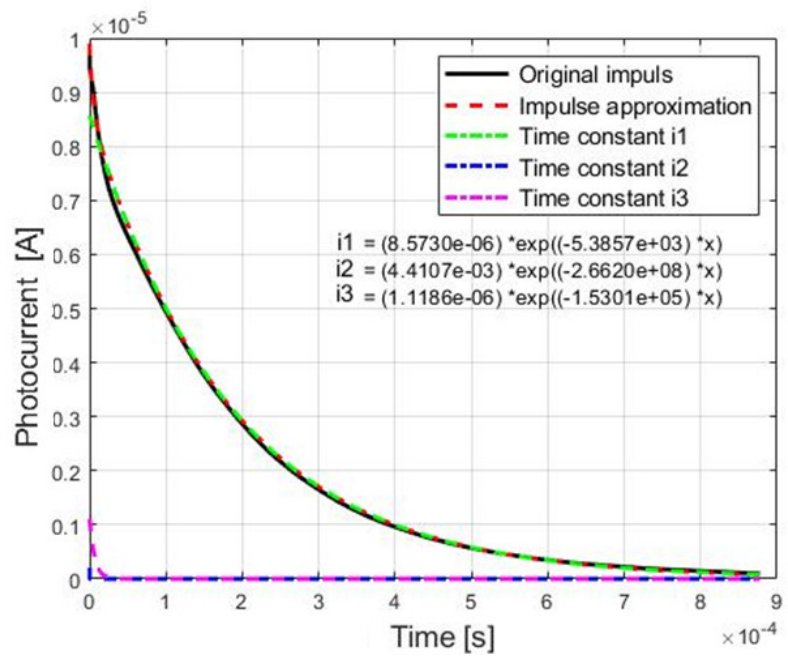

Figure 14. Determined time constants of the photocurrent pulse for the GaP-2 switch in the conduction state with $2.76 \mathrm{eV}$ $(450 \mathrm{~nm})$ photon energy at $7.11 \mathrm{kV}$. The original pulse waveform is marked in black, the numerically determined sum of time constants in red, as green, blue and purple are marked in order time constants i1, i2 and i3

From the characteristics presented in figures 13 and 14 it follows that the first time constant i1 has the greatest impact on the shape and time of the sample entering the blocking state. The second and third time constants (i2 and i3) affect only the maximum current value and reach values close to zero after a short period of time, on the order of 100 ns. Figure 15 presents the impact of photon energy on photocurrent time constant waveforms for the GaP-1 switch. Figure 16 shows a comparison of the determined time constants for the GaP-2 switch for different applied voltages.

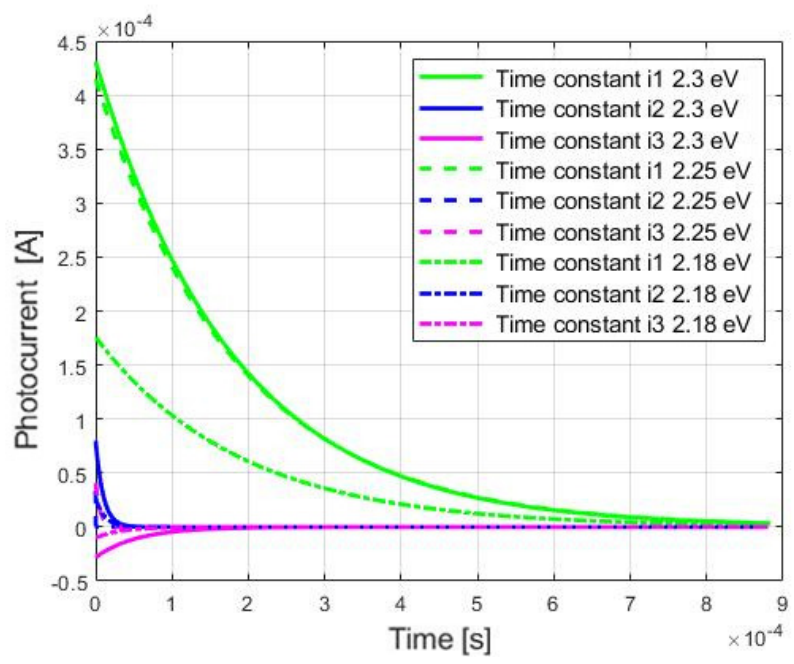

Figure 15. Determined time constants of photocurrent waveforms for the GaP-1 switch in the conduction state with photon energy $2.3,2.25$ and $2.18 \mathrm{eV}$ at $10.2 \mathrm{kV}$. The time constants i1, i2 and i3 for individual photon energies are marked in order with green, blue and purple colors. 


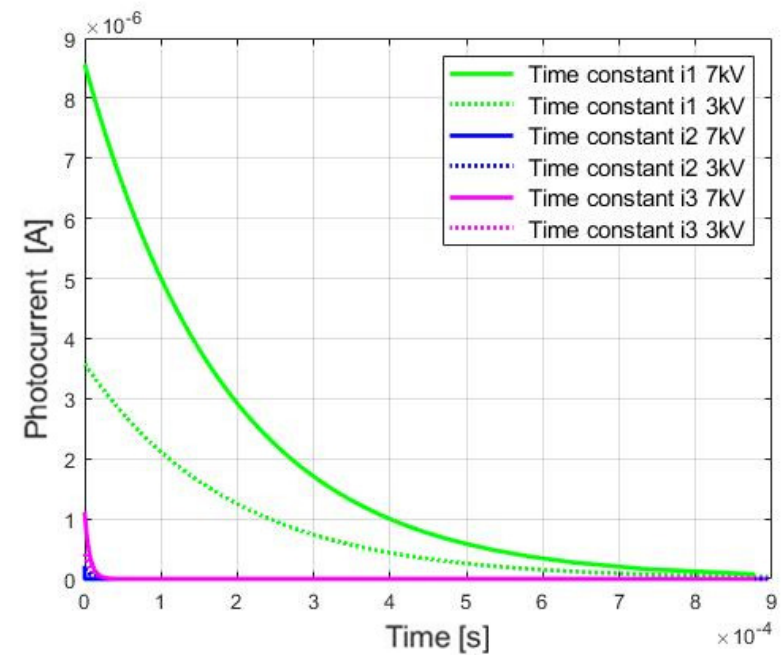

Figure 16. Determined time constants of the photocurrent pulse waveforms for the GaP-2 switch in the conduction state at $2.76 \mathrm{eV}(450 \mathrm{~nm})$ photon energy. The time constants i1, i2 and i3 for 3.06 and $7.11 \mathrm{kV}$ are marked in order with green, blue and purple color

Based on the characteristics presented in figure 15, we can observe that as the photon energy increases, the amplitude of the exponential photocurrent waveform of the time constant il increases. For photon energy smaller than the width of the band gap of gallium phosphide, we can observe a significant decrease in the amplitude of photocurrent waveforms with time constants i1 and i2. At the same time, the decay time of individual photocurrent waveforms does not change. Analyzing figure 16, we can conclude that the maximum values of individual time constants increase as the applied voltage increases.

\section{SUMMARY}

The article presents the results of tests to determine the characteristics of switches made of semi-insulating gallium phosphide in a blocking and conduction state. The time courses of the photocurrent, being a response to the material stimulation by means of an optical impulse generating excess charge carriers, are presented. Time constants of photocurrents were determined. The influence of photon energy on individual components of time constants as well as the photocurrent amplitude achieved at a constant value of applied voltage were illustrated and discussed.

In the blocking state, for GaP-1 and GaP-2 switches, the maximum current achieved was 2500 and $25 \mathrm{nA}$, respectively. In the conduction state, the highest photocurrent values of the GaP-1 sample were recorded for optic pulse length of 540 and $530 \mathrm{~nm}$, corresponding to photon energy of 2.3 and $2.34 \mathrm{eV}$. The obtained photocurrent values were $513 \mu \mathrm{A}$. For the GaP2 sample, the highest photocurrent value of $99 \mu \mathrm{A}$ was observed for a wavelength of $450 \mathrm{~nm}$, while for $700 \mathrm{~nm}$ a photocurrent value of $24 \mu \mathrm{A}$ was achieved. This is due to the fact that the generation of excess charge carriers for photons with energy less than the width of the band gap $(2.26 \mathrm{eV})$ occurs through deep defect centers in the material.

The conductivity in the conduction state for the GaP-1 sample increased by 5 orders of magnitude for optic pulse length of 540 and $530 \mathrm{~nm}$, from $4.9 \cdot 10^{-7}$ to $2.3 \cdot 10^{-2} \Omega^{-1} \mathrm{~cm}^{-1}$. In the case of the GaP-2 sample, an increase in conductivity by 3 orders of magnitude from $6.9 \cdot 10^{-9}$ to $1.98 \cdot 10^{-6} \Omega^{-1} \mathrm{~cm}^{-1}$ was noted. The time of transition to the conduction state and return to the blocking state for both samples did not exceed $900 \mu$ s, which means that these switches can operate at a frequency of $1.11 \mathrm{kHz}$, which can satisfy potential users.

The results of the calculated time constants of the exponential photocurrent waveforms indicate that the first time constant is mainly responsible for the shape of the waveform and the length of time in which the switch returns to the blocking state. The second and third time constants reach values close to zero after about $100 \mathrm{~ns}$. They are responsible for the maximum value of the conducted photocurrent. It was also noticed that with the increase of photon energy near the 
absorption edge for the constant value of the applied voltage, the maximum value of the first time constant increases. As the voltage in the system increases, independent of the excitation wavelength, an increase in the value of the first and second time constant has been noticed.

In the next stage of research, it is planned to increase the tested voltage range above $10 \mathrm{kV}$, as well as to start testing samples of PCSS switches with vertical geometry.

\section{REFERENCES}

[1] Tian, L., Shi, W., "Analysis and operation mechanism of semi-insulating GaAs photoconductive semiconductor switches," J. Appl. Phys., 103, 124512-1-7 (2008).

[2] Shi, W., Tian, L., Liu, Z., Zhang, L., Zhang, Z., Zhou, L., Liu, H. and Xie, W., "30 kV and 3kA semi-insulating GaAs photoconductive semiconductor switch,” Appl. Phys. Lett., 92, 043511-1-3 (2008).

[3] Xu, M., Liu, X., Li, M., Liu, K., Qu, G., Wang, V., Hu, L., Schneider, H., "Transient characteristic of interdigitated GaAs photoconductive semiconductor switch at 1-kHz excitation," IEEE Electron Device Letters, 40(7), 1136-1138 (2019).

[4] Berczyński, R., Kulas, S., J., ”Diagnozowanie dynamiki ruchu styków łączników elektrycznych wysokiego napięcia," Przegląd Elektrotechniczny, 92, 24-28 (2016).

[5] Berczyński, R., Kulas, S., J., "Badanie dynamiki ruchu styków złącznika zwarciowego," Przegląd Elektrotechniczny, 93, 16-20 (2017).

[6] Majda-Zdancewicz, E., Suproniuk M., Pawłowski, M. and Wierzbowski, M., "Current state of photoconductive semiconductor switch engineering," Opto-Electronics Review, 26, 92-102 (2018).

[7] Luan, C., Feng, Y., Huang, Y., Li, H., Li, X., "Research on a novel high-power semi-insulating GaAs photoconductive semiconductor switch," IEEE Transactions on Plasma Science, 44(5), 839-841 (2016)

[8] Kelkar, K. S., Islam, N. E,. Fessler C. M. and Nunnally W. C., "Design and characterization of silicon carbide photoconductive switches for high-field applications," J. Appl. Phys., 100, 124205-1-5 (2006).

[9] Mauch, D., Sullivan, W., Bullick, A., Neuber, A., Dickens, J., "High Power Lateral Silicon Carbide Photoconductive Semiconductor Switches and Investigation of Degradation Mechanisms," IEEE transactions on plasma science, 43(6), 2021-2031 (2015).

[10] Long, H., Jiancang, S.,, Zhenjie, D., Qingsong H. and Xuelin, Y., "Investigation on properties of ultrafast switching in a bulk gallium arsenide avalanche semiconductor switch," J. Appl. Phys., 115, 094503-1-7 (2014).

[11] Suproniuk M., Kamiński, P., Kozłowski, R., Teodorczyk, M., Mirowska, A., Majda-Zdancewicz, E., Wierzbowski, M., Piwowarski, K., Paziewski, P., "Semi-insulating GaP as a material for manufacturing photoconductive semiconductor switches," Proc. SPIE 11055, (2019).

[12] Suproniuk, M., Kamiński, P., Kozłowski, R., Pawłowski, M. and Wierzbowski, M., "Current status of modelling the semi-insulating $4 \mathrm{H}-\mathrm{SiC}$ transient photoconductivity for application to photoconductive switches," Opto-Electronics Review, 25, 171-180 (2017).

[13] Suproniuk, M., Pawłowski, M., Wierzbowski, M., Majda-Zdancewicz, E., Pawłowski, M. K., " Comparison of methods applied in photoinduced transient spectroscopy to determining the defect center parameters: The correlation procedure and the signal analysis based on inverse Laplace transformation," Review of Scientific Instruments, 89, 04470-0044710 (2018). 